\title{
Complexation of Pectins Varying in Overall Charge with Lysozyme in Aqueous Buffered Solutions
}

\author{
Yurij A. Antonov* \\ N.M. Emanuel Institute of Biochemical Physics, Russian Academy of Sciences, Kosygin Str. 4. 119334 \\ Moscow, Russia \\ Miete Celus, Clare Kyomugasho, Marc Hendrickx \\ Centre for Food and Microbial Technology, Faculty of Bioscience Engineering, KU Leuven, Kasteelpark \\ Arenberg 22, Box 2457, B3001 Leuven, Belgium \\ Paula Moldenaers
}

Soft Matter Rheology and Technology, Department of Chemical Engineering, KU Leuven, Celestijnenlaan 200f, Box 2424, B3001 Leuven, Belgium

Ruth Cardinaels Polymer Technology, Department of Mechanical Engineering, TU Eindhoven, Box 513, 5600 MB Eindhoven, The Netherlands

\section{*Corresponding author:Yurij Antonov, e-mail: chehonter@yandex.ru}

ABSTRACT. We examined how a particular structural characteristic of pectin, namely its degree of methoxylation (DM), affects its complexation with lysozyme (lys), whereby the phase behaviour of the complex system as a whole as well as the aggregation state of the complex phase are considered. Low and medium methoxylated pectins bind lys at $\mathrm{pH} 5.1$ and low ionic strength $(I=0.01)$ forming highly concentrated gel like complex particles, whereas highly methoxylated pectins undergo liquid/liquid phase separation with lys. "Mother" pectin with $\mathrm{DM}=95.1 \%$ does not form complexes with lys. Highly charged pectins (low DM) already form complex particles at low pectin/lys weight ratios and have a high yield of the complex phase that consists of relatively large particles. The distribution of pectin within the complex particles has an irregular character for both pectin types. However, whereas for low DM pectin the complex composition reflects that of the mixture, the complex composition with high DM pectin is less sensitive to the mixture composition. Moreover, at low DM, the maximum complexation corresponds to charge neutralization whereas at high DM maximum complexation requires a larger than stoichiometric amount of lys. The minimal $I$ and $\mathrm{pH}$ value at which complexation is completely suppressed, decreases with increasing DM of pectin, indicating the presence of electrostatic interactions in all systems. However, the differences in composition of the complexes and the protein/polysaccharide charge ratio at maximal complexation indicate that the exact binding mechanism depends on the pectin DM.

Keywords: Pectin; lysozyme; complexation; confocal microscopy; structure. 


\section{INTRODUCTION}

Pectin is a natural carboxyl containing linear polysaccharide that is finding increasing applications in the pharmaceutical, biotechnology and food industry on itself and in mixtures with proteins or other polysaccharides (May,1990). The repulsive and attractive forces between pectin and proteins may lead to biopolymer incompatibility (Antonov, Grinberg, Tolstoguzov, 1975, 1979; Antonov, Lashko, Glotova, Malovikova, and Markovich, 1996), depletion flocculation (Antonov, Grinberg, Zhuravskaya, \&Tolstoguzov, 1982) or complex formation (Serov, Antonov, Tolstoguzov, 1985, Tolstoguzov, 1986; 1991). Therefore, at present it is obvious that interactions between pectin and proteins can affect the phase behavior, stability and structure of food systems (Tolstoguzov, 1986). Moreover, these interactions affect the physical properties of food preparations such as their clarity, stability and gel-forming ability (De Kruif \& Tuinier, 2001, Tolstoguzov, 2003). The use of highly methoxylated pectins as flocculants for natural biological materials (defatted milk, milk whey, clover and alfalfa juices) allows to fractionate milk and leaf proteins (Antonov \& Kiknadze, 1987) and to obtain liquid concentrates of micellar casein from defatted milk (Antonov,et al, 1982). Therefore the complexation of pectins with proteins was a subject of intense scientific interest in the last two decades (see for example Girard, Turgeon, Gauthier, 2002,2003a,b; Maroziene, De Kruif, 2000; Zaleska, Ring, Tomasik, 2000; Sperber, Schols, Stuart, Norde, \& Voragen, 2009, 2010; Gilsenan, Richardson, Morris, 2003; Wang, Lee,Wang, \& Huang, 2007; Ru, Wang, Lee, Ding, \& Huang, 2012; Saravanan, 2010; Plashchina, Zhuravleva,\& Antonov, 2007; Bayarri, Oulahal, Degraeve, \& Gharsallaoui 2014; Lin et al. 2015; Amara, Degraeve, Oulahal, \& Gharsallaoui,2017; Schmidt, Cousin, Huchon, Boué, \& Axelos, 2009).

To exploit the potential of pectin complexation for different applications, studying the effects of the structural features of pectin (degree of methoxylation DM, distribution of functional groups along the chain, molecular weight) on its interaction with proteins is very important in food science and technology. It is now generally accepted that electrostatic interactions are the main factor determining complexation of polysaccharides with proteins. Therefore, aggregation of water insoluble complexes is frequently maximal when the charge ratio is equal to 1 , which corresponds to neutralization of the complexes (Ball, et al, 2002; Gummel, Boue, Deme, Cousin, 2006). Nonelectrostatic attractions sometimes promote protein/polysaccharide association and facilitate formation of water insoluble protein/polysaccharide complexes (Carlsson, Linse, \& Malmsten, 2001; Carlsson, Malmsten, \& Linse, 2003). In the case of low degrees of pectin esterification hydrogen bonding may cause a minor contribution to coacervate formation (Girard, Turgeon, \& Gauthier, 2003b). Girard, Turgeon, and Gauthier (2002) demonstrated that interactions in the $\beta$ 
$\mathrm{lg} /$ pectin system are mainly caused by electrostatic forces, and, to a lesser extent, hydrogen bonding. Hydrophobic interactions between pectin chains can be neglected due to the hydrophilic backbone of pectin.

The aim of the present study is to examine how one particular structural characteristic of pectin, namely its DM, affects its interaction and complexation with lysozyme (lys) as well as the structure of the complex particles. To obtain unambiguous results, care was taken to vary only the pectin DM while keeping its Mw constant. Lys is a well-studied $14.3 \mathrm{kDa}$ globular protein with enzymatic activity which has a net positive charge in the $\mathrm{pH}$ range up to its $\mathrm{pI}(10.5)$. We focus on a few important characteristics of complexation namely determination of i) the possible contribution of hydrogen bonding on the interaction, ii) the critical surface charge values above which pectin does not form complexes with lys, iii) the dependence of the aggregation state of the complex phase on $\mathrm{DM}$, and iv) the distribution of the biopolymers within the complex phase for pectins with various DM values. This allows us to determine the mechanism of binding in such systems, which will allow to compare, in future work, the peculiarities of complexation in these systems with those containing pectins with a blockwise distribution of methylesters. Thereto dynamic light scattering, confocal laser scanning microscopy (CLSM), optical and scanning electron microscopy, phase analysis, electrophoretic mobility, and turbidity measurements were utilized.

\section{MATERIALS AND METHODS}

\subsection{Materials}

Biopolymers and reagents. Lys from chicken egg white (dialyzed, lyophilized powder containing $96 \%$ of the main protein) was purchased from Sigma-Aldrich and used without further purification. Pectin with a statistical distribution of methylester groups, and varying DM values namely $16.2 \%$ (P16.2), 27.8\% (P27.8), 38.2\% (P38.2), 66.5\% (P66.5) and 77.7\% (P77.7) were produced through controlled chemical deesterification of a parent citrus pectin with $\mathrm{DM}=95.1 \%$ (P95.1) by titration with predetermined amounts of $0.1 \mathrm{M} \mathrm{NaOH}$ solution (at $4{ }^{\circ} \mathrm{C}$ while maintaining a $\mathrm{pH}$ of 11 ) as described in another study (Celus, et al, 2017). The resulting solutions were adjusted to $\mathrm{pH}$ 6, dialyzed (Spectra/Por ${ }^{\circledR}, \mathrm{MWCO}=12-14 \mathrm{kDa}$ ) for $48 \mathrm{~h}$ against demineralized water and subsequently lyophilized. Measurement of the DM was performed using Fourier transform infrared spectroscopy (FT-IR) (Shimadzu FTIR-8400S, Japan), as in Celus et al. (2017). The average molar mass of the samples, as determined by HPSEC chromatography was $44.6 \pm 3.5 \mathrm{kDa}$ and varied between $41.1 \mathrm{kDa}$ and $48.1 \mathrm{kDa}$ whereas the mother pectin had a molar mass of $56.1 \mathrm{kDa}$ (Celus et al., 2017). All other reagents were of analytical grade. Milli-Q ultrapure water was used in all experiments. 
Preparation of the protein and polysaccharide solutions and protein/polysaccharide mixtures. To prepare solutions of lys and pectin with the required concentrations, the weighed amount of biopolymer was gradually added to an acetic acid-sodium acetate buffer $(\mathrm{pH} 5.1, I=0.01)$ at $23^{\circ} \mathrm{C}$ and stirred for $1 \mathrm{~h}$ at this temperature. The resulting solutions were centrifuged at $50000 \mathrm{~g}$ and $23^{\circ} \mathrm{C}$ for $1 \mathrm{~h}$ to remove insoluble particles. The lys content in the stock solution was determined by means of UV absorption using the extinction coefficient for highly purified lysozyme which is $2.64 \mathrm{ml} \mathrm{mg}^{-1} \mathrm{~cm}^{-1}$ at $281.5 \mathrm{~nm}$ (Aune, \& Tanford, 1969). Concentrations of pectin in the stock solutions were determined by drying to constant weight at $104^{\circ} \mathrm{C}$. Solutions of the biopolymers were kept at least overnight in the fridge to allow for full hydration of the molecules. To prepare mixed solutions of lys with pectin with the required concentrations, weighed amounts of the pectin stock solution were added to the lys stock solution at $23^{\circ} \mathrm{C}$. To study the effect of $\mathrm{pH}$ on the complexation behavior, the $\mathrm{pH}$ of the complex mixtures was changed in a range from 5.0 to 11.0 by addition of $0.1-0.5 \mathrm{M} \mathrm{NaOH}$.

\subsection{Methods}

\subsubsection{Turbidity measurements}

Turbidity values of the systems as function of the pectin/lys weight ratio (q) were measured at 500 $\mathrm{nm}$ using a Unico SQ2800 UV/VIS spectrometer. Solutions were mixed at $23^{\circ} \mathrm{C}$ for 90 minutes to reach steady state conditions. The error of the turbidity measurements is typically about $2 \%-3 \%$; in the weight ratio range from 0.2 to 0.8 the errors were markedly larger (6-8\%). With increasing q, the complexation behavior underwent different transitions characterized by qonset and qset, corresponding to the transitions from the absence of complexation to the formation of water soluble complexes and vice versa, as well as $\mathrm{q}_{\phi}$ and $\mathrm{q}_{\phi}{ }^{*}$, corresponding to the transitions from water soluble complexes to water insoluble complexes and their phase separation and vice versa

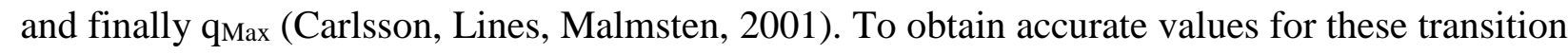
points, additional characterizations were performed. The qonset value was determined by turbidimetric titration as indicated in Fig 1a'. These results were confirmed by dynamic light scattering (DLS) characterization, in which the average size of the complexes exceeded that of pectin by $10 \%$ at qonset. The qset value was the minimum q value for which the turbidity value of the pectin/lys mixture became equal to that of a pectin solution with the same pectin concentration as in the mixture. The $\mathrm{q}_{\phi}$ and $\mathrm{q}_{\phi} *$ values were determined as the minimum and maximum $\mathrm{q}$ values at which the turbidity increased with time, which was quantified as an increase of $>2 \%$ during 15 min under quiescent conditions for samples that had been stirred for $30 \mathrm{~min}$ before the test.

\subsubsection{Phase analysis}


The yields of lysozyme (Y) in the complex phase and the supernatant were determined after separation of the complex pectin/lysozyme system by centrifugation at $10000 \mathrm{~g}$ and $23^{\circ} \mathrm{C}$ for 20 min. The masses of the complex phase and supernatant were measured as well as the concentration of lysozyme in the supernatant. The latter was determined by measuring the absorption value at $280 \mathrm{~nm}$ at $I=0.5$. The absorbance of free lysozyme in a $0.5 \mathrm{M} \mathrm{NaCl}$ solution of $1 \%$ concentration was $A_{1 \%, 280}=27.21$. This allowed to calculate the yields. The experimental errors were approximately 6-8\%.

\subsubsection{Electrophoretic Mobility}

s-potential measurements of pectin, lys and pectin/lys complexes at different $q$ values were performed at $23^{\circ} \mathrm{C}$ with a 90 Plus particle size analyzer (Brookhaven instruments Inc.) using a rectangular quartz capillary cell. For each sample the $\varsigma$-potential was determined at least ten times and the average value is reported.

\subsubsection{Dynamic light Scattering}

Determination of the intensity size distribution functions of pectin and lys solutions as well as pectin/lys mixtures was performed by means of DLS with an ALV/CGS-3 compact goniometer system (ALV GmbH, Germany). The procedure has been described elsewhere (Antonov, Moldenaers, \& Cardinaels, 2017).

\subsubsection{Scanning electron microscopy (SEM).}

Microstructural investigation was performed with a scanning electron microscope JEOL JSM-840. Pectin/lys mixtures at desirable concentrations were subjected to complete drying at $23^{\circ} \mathrm{C}$ using a graphene plate. The SEM images were recorded multiple times and on multiple samples to ensure that representative images are obtained. Images were obtained less than 5 min after introducing the sample in the chamber.

\subsubsection{Brightfield imaging}

Microscopy observations were performed with an Olympus BX51W1 fixed stage microscope equipped with a high resolution CCD-camera, (1000x1000 pixels, C-8800-21, Hamamatsu) using objectives with magnifications 5,10, 20, and 50 times.

\subsubsection{Fluorescent imaging}

Fluorescent imaging was performed using a multi-beam confocal microscope (VisiTech, UK), equipped with an oil-immersion objective (20x, 0.85 NA, Olympus, Japan) using $532 \mathrm{~nm}$ and $642 \mathrm{~nm}$ as excitation wavelengths. Before imaging, pectin and lys were fluorescently labeled by storing a pectin solution containing Atto $647 \mathrm{~N}$ dye (ATTO Tec. Germany) at $5^{\circ} \mathrm{C}$ for 3 days, and a lys solution containing Rhodamine B dye was kept under the same conditions. This labeling allowed the signal from Lys (red) and pectin (green) to be resolved. Image analysis was performed 
using ImageJ v1.43r software.

3. Results and discussion.

\subsection{Characterization of complexation at $\mathrm{pH} 5.1, I=0.01$ and $23^{\circ} \mathrm{C}$}

\subsubsection{The main parameters of complexation and zeta potentials.}

The $\varsigma$-potential of the pectins with various DM decreases linearly with DM, ranging from -34.1 $\mathrm{mV}$ for P16.2 to $-2.8 \mathrm{mV}$ for P95.1 (data not shown). At $\mathrm{pH} \mathrm{5.1,} \mathrm{lys} \mathrm{was} \mathrm{found} \mathrm{to} \mathrm{have} \mathrm{a} \mathrm{net}$ positive $\varsigma$-potential of $+7.7 \mathrm{mV}$, which is close to literature data (Kuehner, Engmann, Fergg, Wernick, Blanch and Prausnitz, 1999; Sophianopoulos and Vanholde, 1961).Therefore, based on the opposite charge of the two polymers, they are expected to exhibit attractive interactions. Mixing transparent solutions of lys and pectin led to a quick appearance of turbidity if the pectin/lys weight ratio (q) exceeded the qOnset value. Figure 1a-c shows the turbidity values at 500 $\mathrm{nm}\left(\tau_{500}\right)$ as a function of $\mathrm{q}$ for complex pectin/lys systems containing P16.2, P66.5 or P77.7. It was found that the complex systems containing P95.1 remained transparent for all q values studied (from $10^{-4}$ to 100 ). Based on turbidity as a function of the pectin/lys weight ratio $\mathrm{q}$, the transitions in the state of the mixed system between miscible, soluble complex, and insoluble complex can be pinpointed (Figs 1a-c, region I-V).

\section{Figure 1}

The mixture behavior was clearly dependent on the DM of pectin and the pectin/lys weight ratio (q), and consequently, on the charge ratio of the two biopolymers. The dependence of $\tau_{500}$ on $q$ has an extremal character with the maximum $\tau_{500}$ occurring at $\mathrm{q}_{\mathrm{Max}}=0.21$ for the P16.2/lys systems, $\mathrm{q}=0.24$ for the P38.2/lys systems (data not shown), $\mathrm{q}=0.30$ for P66.5/lys systems and $\mathrm{q}=0.51$ for P77.7/lys systems respectively. Taking into account that the weight average molecular weight of Lys is $14.3 \mathrm{kDa}$ (Rezwan, Meier, Gauckler, 2005) and that of pectin is on average 44.6 $\mathrm{kDa}$, we can roughly evaluate the "molar" ratio pectin/lys in the complex phase. A simple calculation showed that this ratio was $\sim 18: 1$ mole lys/mole pectin for the P16.2/lys systems, 15:1 for the P38.2/lys systems, 12:1 and 7:1 for the P66.5/lys and P77.7/lys systems respectively. The qMax values as a function of DM are presented in Fig. 2a. At relatively low DM values, these ratios increase linearly with DM, and subsequently change more sharply at DM $>66 \%$. To assess the contribution of electrostatic interactions to the complexation process, we compared the $\mathrm{q}_{\mathrm{Max}}$ values for P16.2/lys and P66.5/lys systems determined from the data of the turbidimetric titration with those calculated on the basis of the charge ratio of the cationic groups of lys and 
$44600 \mathrm{Da}$ and the molecular weight of galacturonic acid is $194 \mathrm{Da}$. Formation of the glycosidic bond in pectin involves formal elimination of a molecule of water, thereby reducing the mass per residue by $18 \mathrm{Da}$, and conversion of the uncharged $\mathrm{COOH}$ group to the charged carboxylate form gives a further reduction of $1 \mathrm{D}$. Moreover, for esterified residues, the mass of the methyl group causes an increase of $15 \mathrm{Da}$. Thus the effective values of the molecular weight are $175 \mathrm{Da}$ for charged galacturonate residues and $190 \mathrm{Da}$ for the esterified form. Accordingly, the amount of galacturonic acid units is equal to 254.8 (44600:175) for charged pectin, and 234.7 (44600:190) for the esterified form of pectin. Taking into account that the content of the galacturonic acid in pectin samples is 85\% (Ngouémazong et al. 2011), and the degree of dissociation of the galacturonic acid at the given conditions is 0.97 (Kohn, 1973) we obtained 176.0 negative charges per mole for P16.2 and 64.8 negative charges per mole for P66.5. On the other hand, one mole of 100\% lys contains 129 amino acid residues (or 116.1 amino acid residues in our sample containing 90\% protein) and includes positively charged lysine (3.97 charges), arginine (8.86 charges) and histidine (0.75 charges), according to Levis, Snell, Hirschmann, and Fraenkel-Conrat (1950), i.e. 13.5 positive charges per mole. In that way, the calculated protein/polysaccharide molar ratio within a complex in the case of purely electrostatic interactions is 18.8 for the P16.2/lys system. The molar ratio obtained was equal to that obtained from turbidimetry, which was 18 mole lys/mole pectin. The estimated lys/pectin molar ratio for the P66.5/lys system was 4.8 . The lys/pectin molar ratio for the P66.5/lys system calculated from the molecular structure of the biopolymers was noticeably lower than that determined by turbidimetry (12.1 mole lys/mole pectin) which may be due to some aggregation of lys within the P66.5/lys complex particles.

The qonset and $\mathrm{q}_{\phi}$ values for P16.2/lys systems were very small $\left(5 \cdot 10^{-4}\right.$ and $\left.2 \cdot 10^{-3}\right)$, as shown in Fig 2a,b but they increased significantly with $\mathrm{DM}$, especially for pectin with the highest $\mathrm{DM}=77.7 \%$. This indicated that lys interacts with pectins to form water insoluble complexes starting from extremely low concentrations of the latter except for pectin with the highest DM. Deflection of the linear dependence at the highest DM values may be due to the fact that the binding constant approaches the critical value at which macromolecular complexes do not form. At DM $=95.1 \%$ associative phase separation did not occur at all, at least up to total mixture concentrations of 8 $\mathrm{wt} \%$. Fig $2 \mathrm{~b}$ summarizes the phase behaviour of all systems studied, and the liquid or solid state of the complex phase. As can be seen in Fig $2 \mathrm{~b}$ formation of water soluble complexes observed only in a very narrow q range for all DM values studied (excluding DM 95.1\%). Formation of complex coacervates took place at $\mathrm{q}_{\phi}$ values equal and higher than 0.01 for systems containing highly methoxylated pectins (P66.5 and P77.7), and a single-phase state was typical for the P95.1/lys system for all q values. 
The $\varsigma$-potential of the complex pectin/lys systems as a function of the pectin/lys weight ratio (q) at different DM values is shown in Figure 2e. The positive charge of lys was rapidly neutralized after adding pectin, and the surface charge of the formed complexes turned into negative values at higher $\mathrm{q}$ ratios. Complete neutralization of the positive charge took place at $\mathrm{q}=0.21,0.24,0.3$ and 0.51 for systems containing P16.2, P38.2, P66.5 and P77.7 i.e. at the q ratio that corresponds to maximum complexation according to the turbidity values (Fig. 1a-c). With knowledge of the net surface charge of lys and pectin ( $\varsigma_{\text {Pectin }}$ and $\varsigma_{\text {Lys }}$ ) and their weight ratio in the complex, the estimated total charge of the complex becomes:

254

255

256

257

258

259

260

261

262

Hence, for P16.2/Lys systems a net charge of zero is expected at $q=0.22$, which corresponds well to the actual measured $\zeta$-potential of the complex as well as to the q value of maximum complexation. Similar results were obtained for P38.2/Lys and P66.6/Lys systems. Only for P77.7/Lys systems, a significant difference between the expected transition from positive to negative charge and the actual one, was observed. The results obtained indicated first that electrostatic forces were the main driving forces for complexation of lys with pectins having a DM from $16 \%$ to $66.5 \%$ and additional forces play a role at higher DM. This is in agreement with the direct calculation of the charges in lysozyme and pectin as indicated by the turbidity analysis. Besides, we established that the mother pectin with DM 95\% having a zeta potential value of -2.8 $\mathrm{mV}$ did not form water insoluble complexes with lys, whereas P77.7 having a zeta potential value of $-9.95 \mathrm{mV}$ formed water insoluble complexes with lys. Hence, it can be suggested that the minimal zeta potential values of pectin at which insoluble complexes with lys begin to form is on the order of $-6 \mathrm{mV}$.

3.1.2. Effect of DM on the aggregation state and yield of the complex phase, the size and morphology of the complex particles.

In order to quantitatively evaluate the degree of complexation for pectins with different DM values the complexation process was characterized by means of phase analysis. Figures 3a-c and Figure 4a present the yield of lys (mass percentage) in the biopolymer rich complex phase and supernatant as a function of the weight ratio $q$ for the mixtures containing P16.2 (Fig. 3a), P38.2 (Fig. 3b), P66.5 (Fig. 3c) and P77.7 (Fig. 4a). The maximum yields of lys in the complex phase decreased from $99 \%$ for the system containing P16.2 to 58\% for the system with P77.7. These maximum 
yields were observed at q values very similar to those established by turbidimetry measurements (Fig. 1). The maximum yield of the water insoluble complex ( $\left.\mathrm{Y}_{\max }\right)$ as a function of DM is shown in Fig. 4b. Extrapolation of the curve to zero $Y_{\max }$, gives a DM value of $87 \pm 2 \%$. At this DM value of pectin water insoluble complexes are not formed.

\section{Figure 3}

\section{Figure 4}

Brightfield microscopy images of complex pectin/lys systems containing pectins with various DM values are illustrated in Figure 5 a-d. Complex particles formed on the basis of P16.2 and P38.2 were similar in morphology. They were solid and had an irregular form (Fig. 5a,c). At constant q value, their size increased considerably when the concentration of lys was increased from $0.04 \mathrm{wt} \%$ to $4 \mathrm{wt} \%$ (Fig. 5a,c). In contrast to the above mentioned systems, mixtures of lys with P66.5 (Fig. 5b,d) or P77.7 (data not presented) formed complex particles with a regular form close to spherical. Centrifugation of these complex systems showed that both their coexisting phases have a liquid state of matter, which is different from P16.2/lys and P38.2/lys systems. The concentrated phase of the latter systems has a solid state of matter. In other words, the systems containing lys and P66.5 or P77.7 were coacervates. Transition from solid complex particles to coacervates was probably caused by the considerable decrease of the overall charge of pectin, which significantly suppresses interactions leading to an increased mobility in the complexes, possibly due to differences in the conformation of the molecules. Complex coacervation is usually observed in systems containing proteins and weak polyelectrolytes, such as for example arabic gum (De Kruif, \& Tuinier 2001) and highly methoxylated pectin (Schmidt et al., 2009). However, the more than linear increase of $\mathrm{q}_{\text {onset }}$ and $\mathrm{q}_{\phi}$ with DM at high DM (Fig. 2b) suggests that at low charge density, the interactions were suppressed more than would be expected based purely on charge density. A nucleus of the complex phase for P66.5/lys systems was formed already at a concentration of lys as low as $10^{-5} \mathrm{wt} \%$ and could more easily be visualized by SEM due to limited aggregation (Figure 5e). The image presented in Fig. 5e shows a nucleus of the complex phase (arrow A), small associates of nuclei of the complex phase (arrow B in Fig. 5e), as well as large complex associates, which are the precursors of droplet formation (arrow $\mathrm{C}$ in Fig. 5e).

\section{Figure 5}

The sizes of the complex particles at different $\mathrm{q}$ values were characterized by means of DLS measurements. The data obtained are presented in Figures $6 a, b$ and $7 a-c$. In the binary solutions, all pectins were present as associates with sizes ranging from 104 to $114 \mathrm{~nm}$, depending on DM (Fig. 6a-e). The average radius of the lys monomer was $1.9 \mathrm{~nm}$ (data not shown), in 
accordance with literature (Parmar,\& Muschol, 2009; Valstar, Brown, Almgren, 1999). DLS data obtained for the systems containing pectins with different DM showed both common features as well as significant distinctions. As can be seen in Figs. 6a-b and 7a-c, for all systems studied the presence of even a very small amount of pectin in the lys solution (at q=0.05), led to a shift of the dominant peak to a larger average particle radius although the absolute particle size was dependent on the DM of pectin. A maximal size $(2030 \mathrm{~nm})$ was obtained with P16.2, and it decreased with DM to $419 \mathrm{~nm}$ for P66.5. Complex particles formed in P66.5/lys systems were characterized by a lower polydispersity and size as compared to those in the other two systems. A similar behavior was seen for the P77.7/lys system (data not presented). The dependence of the scattering intensity of all systems on $\mathrm{q}$ has an extremal character with the maximum ( $\mathrm{q}_{\mathrm{Max}}$ ) corresponding to the value found by other techniques (Figs. 1a-c). When q increased further above $\mathrm{q}_{\mathrm{Max}}$, the size of the complex particles became very similar to that of free pectin. The average size of the P95.1/lys system was the same as that of P95.1 at the corresponding concentrations (data not presented). Therefore we can state that pectin with DM 95.1\% does not form either water soluble nor water insoluble complexes.

\section{Figure 6}

Figure 7

\subsection{Ionic strength and $\mathrm{pH}$ stability of complexes}

Figure 8a presents $\tau_{500}$ values of complex pectin/lys systems as a function of the $\mathrm{pH}$ obtained for pectins with various DM values. All plots were obtained at q values corresponding to qMax at $I=0.01$ and $\mathrm{pH} 5.1$ for the different systems. A first type of behavior can be observed when changing the $\mathrm{pH}$ from $\sim 5.2$ to 6.2 . In this range pectin molecules become completely ionized at $\mathrm{pH}$ values above and equal to 6 (Kohn, 1973), whereas lys molecules were completely ionized. As can be seen in Fig. 8a, complexation of pectin with lys depended strongly on the dissociation degree of the pectin molecules both for the highly charged and lowly charged pectin samples. In the $\mathrm{pH}$ range from 6.5 to 10 the turbidity of the systems with highly charged pectins changed weakly, whereas the turbidity values of the systems with lowly charged pectins changed dramatically. Taking into account that in the given $\mathrm{pH}$ range the carboxyl groups of pectins were completely ionized (Kohn, 1973) and the positive charge of lys decreases monotonically up to $\mathrm{pH}=9-10$ (Bharti, 2014) we can state that in the given $\mathrm{pH}$ range the complexation of the highly charged pectin sample (P16.2) depended weakly on the net charge of lys whereas complexation of highly methoxylated pectins 
depended strongly on the net charge of lys. With increasing $\mathrm{pH}$ from 10 to 10.5 the net charge of lys decreased sharply and became zero at pH 10.5 (Bharti, 2014). Therefore complexation of lys with pectin was suppressed even for the highly charged pectin. However, the minimal $\mathrm{pH}$ value at which $\tau_{500}$ becomes zero $\left(\mathrm{pH}_{\mathrm{set}}\right)$, indicating the absence of complexation, decreased linearly with the DM of pectin. The absence of complexation in the vicinity of the isoelectric of lys clearly confirms the dominant contribution of electrostatic intermacromolecular interactions whereby other interactions are not strong enough to lead to measurable complexation.

\section{Figure 8}

The $\tau_{500}$ values of the pectin/lys systems as a function of $I$ at different DM values are presented in Fig. 8b. For the highly charged P16.2 and P38.2 this dependency has a nonmonotonic character displaying a maximum in complex formation at $I \approx 0.03$. Similar nonmonotonic dependencies have been observed for other protein/polysaccharide and protein/polyelectrolyte systems in which the protein contains charged patches. This nonmonotonic dependency on the salt concentration has been attributed to the counteracting effects of salt in screening repulsions as well as weakening attractions (Antonov, Zhuravleva, Cardinaels, \& Moldenaers, 2018; Seyrek, Dubin, Tribet, \& Gamble, 2003). The minimal $I$ values above which complexation was suppressed depended on the overall charge of pectin, which confirmed the electrostatic character of the complexation. A reason for the absence of a nonmonotonic dependance of $\tau_{500}$ on $I$ for systems with highly methoxylated P66.5 and P77.7 can be the presence of very weak short-range attractive interactions that screen repulsions between macroions. The dependence of $I_{\text {set }}$ of the systems on DM of pectin is presented in the inset of Fig. 8b. The $I_{\text {set }}$ values decreased with increasing DM, especially at DM higher than $60 \%$. Deflection of the dependence at the highest DM values may be due to the fact that the binding constant approaches the critical value at which macromolecular complexes do not form. Thus, we can suggest that the effect of DM on $I_{\text {set }}$ values for pectins with low methoxylation degree was similar to that observed for the dependences of q Onset on DM, and $\mathrm{q}_{\phi}$ on DM (Fig 2b). Complete suppression of complexation at specific $I$ values in pectin/lys systems even for the highly methoxylated pectins, strongly suggests that the role of hydrogen bonds in the complexation was insignificant.

\subsection{Protein distribution within the complexes.}

CLSM was applied to monitor the distribution of pectin and lys within pectin/lys complexes at different q values, and to establish possible effects of the overall charge of pectin on this distribution. The results obtained are presented in Figs. 9 and 10. For the systems containing low 
and medium methoxylated pectins six characteristic compositions of the complex system were considered (Fig. 9a-l) namely $\mathrm{q}=0.05$, corresponding to an excess of lys, $\mathrm{q}=\mathrm{q}_{\text {Max }}$, corresponding to maximal yield of the complex according to the phase analysis and turbidity measurements (Figs. 1a-c, 3a-c, 4a), q=0.5 and 0.9, and two compositions characterized by an excess of pectin in the complex system $(q=1.5$ and 2.0). It can be seen that the intensity signal from pectin and lys was dependent on the pectin/lys weight ratio in the complex system. At $\mathrm{q} \leq \mathrm{q}_{\mathrm{Max}}$ the intensity signal from the positively charged lys protein (red) was dominant (Fig. 9a, b, g, h) whereas at q>qMax the intensity contribution from pectin (green) became dominant in the complex particles (Figs. 9c-f, and 9i-1). This indicates that a higher content of pectin in the complex mixture results in a higher pectin content in the complex phase. At $\mathrm{q}>\mathrm{q}_{\mathrm{Max}}$, the yellow signal corresponding to mutual compensation of macro counterions of lys and pectin was also present. However, the distribution of pectin within the complex particles has an irregular character with zones of compensated charges coexisting with zones that consist predominantly of either pectin or lys. In systems containing P66.5 at q> q Max the intensity contribution from pectin (green) in the complex particles changed insignificantly with the q values (Fig. 10b-d). This suggests that there is a difference in the binding mechanism of lys with lowly methoxylated and highly methoxylated pectins. Besides, there were small regions inside the complex particles with an excess of fluorescence intensity from one of the biopolymers indicating an irregular distribution of charge within the complex particles.

\section{Figure 9}

\section{Figure 10}

\subsection{Complexation mechanism}

In general, there are two different ways for binding macromolecular ligands to an acceptor molecule (Figure 11a,b). Both mechanisms differ in the reactivity of free and partially covered acceptor chains. CLSM analysis of the P16.2/lys and P38.2/lys systems (Fig. 9) showed that the pectin/lys weight ratio within the complex particles increased with the pectin content in the complex pectin/lys systems. In other words, the composition of such particles depended on the composition of the initial mixture. This implies that the reactivity of free pectin chains may be considered higher than that of pectin partially covered by lysozyme. This is schematically depicted in Fig. 11a variant 2. The other mechanism is the so-called "all or none" type complex formation mechanism established by Michaels, Mir and Schneider (1965a,b) for oppositely charged polyelectrolyte systems. If the interaction of P16.2 and P38.2 with lys would follow an "all or none" type complex formation mechanism (Fig. 11a variant 1), then the reactivity of the pectin 
chain partially covered by lysozyme should be considered higher than that of free pectin chains, and most pectin chains in the complex phase would interact with lys to full neutralization, resulting in a uniform yellow color on the confocal microscopy images, which is not the case.

\section{Figure 11}

Combining the CLSM and DLS data allows to propose a schematic structure of the complexes. Due to charge neutralization at $\mathrm{q} \leq \mathrm{q}_{\mathrm{Max}}$, interpolymer complexes of P16.2/lys and P38.2/lys systems exhibit reduced solubility and thus associate forming large aggregates, in accordance with the complexation mechanism proposed by Dubin and coworkers (Tsuboi et al.,1996) and Takahashi et al (Takahashi et al., 2000). Based on the radius values of the P16.2/lys complexes at $\mathrm{q}=0.21$ (i.e. under conditions of maximal yield of the complex phase when the molar ratio of lys and P16.2 in the complexes is $~ 17: 1$ ), the number of lys chains and pectin molecules comprising the complexes can be approximated. As depicted schematically in the left plot of Fig. 11b, each complex particle consists of several hundred pectin chains and several thousand lys molecules. At q > qMax, the number of lys molecules interacting with each P16.2 or P38.2 chain decreases when more pectin is present, leading to limited charge neutralization, which hinders the aggregation of the complexes. Therefore, the formed complexes consist of decreasing total numbers of pectin chains and protein molecules, and a relatively higher content of pectin in the complex precipitate.

The DLS results for the P66.5/lys system showed that the final sizes of the complex particles are much smaller as compared to those in mixtures containing low esterified pectin (Figs. 6 and 7). Based on the CLSM data (Fig. 10) it is logical to suggest that the composition of the complex particles of the P66.5/lys system depends weakly on the composition of the initial mixture, due to weaker electrostatic interactions of lys with P66.5 (formation of negatively charged complexes is difficult due to the low charge of the P66.5 molecules). The gradual increase of the pectin/lys ratio in the complex phase indicated that the interaction mechanism was intermediate between the "all or none" and the other interaction mechanism, with neither free pectin chains nor partially neutralized ones having a dominantly high reactivity. Therefore almost completely neutralized small complex particles and completely free P66.5 can coexist in such systems. This is schematically depicted in the right plot of Fig. 11b.

\section{Conclusion}

The overall results demonstrated that the phase state (one- or two-phasic), structure, and morphology of pectin/lys systems were dependent on the DM of pectin. Mixtures of lys with P16.2 or P38.2 formed a suspension of solid complex particles, whereas the presence of P66.5 in a lys 
solution led to the formation of an emulsion (complex coacervation). The interaction has a dominantly electrostatic character, whereby the role of hydrogen bonds in complexation appeared insignificant. Formation of water soluble complexes took place for these systems only in a very narrow q range. A single-phase state of the system without formation of water soluble complexes was observed only in the presence of P95.1 having a zeta potential value equal to $-2.71 \mathrm{mV}$.

The net charges of pectin and lys have different effects on complexation depending on the $\mathrm{pH}$ range. In the $\mathrm{pH}$ range from 5.2 to 6.2 complexation of lys with both lowly and highly charged pectins was determined mainly by the ID of the pectin molecules. On the other hand, in the $\mathrm{pH}$ range from 6.5 to 10 complexation of lys with highly charged pectins (P16.2, P38.2) depended weakly on the ID of lys, whereas complexation of lys with lowly charged (highly methoxylated) pectins was more sensitive to the ID of lys. The $\mathrm{pH}_{\text {Set }}$ values decreased linearly with the DM values of pectin starting from $\mathrm{pH}_{\text {Set }}=10.99$ for the system with $\mathrm{P} 16.2$ to $\mathrm{pH}_{\text {Set }}=8.89$ for the system with P77.7.

The binding mechanism of P16.2 and P38.2 with lys was different from that of P66.5 or P77.7 with lys. In the case of the system containing highly charged pectin the number of lys molecules interacting with each pectin chain decreased when more pectin was present, leading to limited charge neutralization, which hinders the aggregation of the complexes. Therefore, the formed complexes contained decreasing total numbers of pectin chains and protein molecules, and a relatively higher content of pectin in the complex precipitate. This implies that the reactivity of free pectin chains may be considered higher than that of pectin partially covered by lys. On the other hand, in the case of the system containing lowly charged pectin the composition of the complex particles depended weakly on the composition of the initial mixture. Neither free pectin chains nor partially neutralized ones have a dominantly high reactivity.

Conclusions obtained from the present study are a stepping stone for further comparison with the complexation of pectin/lys systems containing pectin with similar DM values and a blockwise distribution of the charged carboxyl groups along the polysaccharide chain. Understanding the effect of the overall charge of the pectin molecules as well as its distribution along the pectin chain on its complexation with protein is important for the prediction of the stability of food structures in pectin/protein systems.

\section{Highlights (for review)}

Complexation of pectin with lys was observed only at DM less $95 \%$.

P66.5 and P77.7 formed liquid complex coacervates with lys.

$$
\text { In the } \mathrm{pH} \text { range from } \sim 5.2 \text { to } 6.2 \text { complexation depended mainly on ID of pectins. }
$$


Effect of $I$ on complexation of highly charged pectin was not monotonic.

Mechanism of binding was different for low and highly charged pectins.

ACKNOWLEDGMENT

483

Y.A. Antonov is grateful to KU Leuven for financial support from the Soft Matter Rheology and Technology group.

\section{References}

Amara, C.B., Degraeve, P., Oulahal, N.,\& Gharsallaoui, A. (2017). pH-dependent complexation of lysozyme with low methoxyl (LM) pectin. Food Chem., 236, 127-133.

Antonov, Y.A., Grinberg, W.Y., \& Tolstoguzov, W.B. (1975). Phasengleishgewichte in Wasser/ Elweiß/Polysaccarid-Systemen. 1. Systeme wasser/casein/saures/polysaccharide. Die Starke, $27,424-431$.

Antonov, Y.A., Lozinskaya, N.V., Grinberg, V.Y., Dianova, V.T., \& Tolstoguzov, V.B. (1979). Phase equilibria in water-protein-polysaccharide system. III. Water-soy bean globulinpolysaccharide systems. Colloid and polymer Sci., 257, 1159-1171.

Antonov, Y.A., Grinberg, V.Y.,Zhuravskaya \& Tolstoguzov, V.B. (1982). Concentration of the proteins of skimmed milk by membraneless isobaric osmosis. Carbohydrate polymers, 2, 81-90. Antonov, Y.A., \& Kiknadze, E.V. (1987). Concentration of a protein by polysaccharide. Die Nahrung, 31, 57-61.

Antonov, Y.A., Lashko. N.P., Glotova, Y.K., Malovikova, A., \& Markovich, O. (1996). Effect of the structural features of pectins and alginates on their thermodynamic compatibility with gelatin in aqueous media. Food Hydrocolloids, 10, 1-9.

Antonov, Y.A., Moldenaers, P., \& Cardinaels, R. (2017). Complexation of lysozyme with sodium caseinate and micellar casein in aqueous buffered solutions. Food Hydrocolloids, 62, 102-118.

Antonov, Y.A., Zhuravleva, I.L., Cardinaels, R., \& Moldenaers, P. (2018). Macromolecular complexes of lysozyme with kappa carrageenan. Food Hydrocolloids, 74, 227-238.

Aune, K.C., \& Tanford, C. (1969). Thermodynamics of the denaturation of lysozyme by guanidine hydrochloride. I. Depdendence on $\mathrm{pH}$ at 25 degrees. Biochemistry. 8(11), 45794585 . 
Ball, V., Winterhalter, M., Schwinte, P., Lavalle, Ph., Voegel, J.-C., Schaaf, P.(2002). Complexation mechanism of bovine serum albumin and poly(allylamine hydrochloride) J. Phys.Chem. B, 106, 2357-2364.

Bayarri, M., Oulahal, N., Degraeve, P.,\& Gharsallaoui, A. (2014). Properties of lysozyme/low methoxyl (LM) pectin complexes for antimicrobial edible food packaging. J. Food Eng., 131, $18-25$.

Bharti, B. (2014). Adsorption, Aggregation and Structure Formation in Systems of Charged Particles: From Colloidal to Supracolloidal Assembly. Springer, 2014, Heidelberg, New York, London, Dorfrecht, 150p.

Carlsson, F., Lines, P., \& Malmsten, M. (2001). Monte Carlo simulations of polyelectrolyteprotein complexation. J. Phys. Chem. B, 105, 9040-9049.

Celus, M., Kyomugasho, C., Kermani, Z. J., Roggen, K., Van Loey, A. M., Grauwet, T., \& Hendrickx, M. E. (2017). Fe2+adsorption on citrus pectin is influenced by the degree and pattern of methylesterification. Food Hydrocolloids, 73, 101-109.

De Kruif, C. G., \& Tuinier, R. (2001). Polysaccharide protein interactions. Food Hydrocolloids, $15,555-563$.

Gilsenan, P.M., Richardson, R.K., \& Morris, E.R. (2003). Associative and segregative interactions between gelatin and low-methoxy pectin: Part 1. Associative interactions in the absence of $\mathrm{Ca}^{2+}$. Food Hydrocolloids, 17, 723-737.

Girard, M., Turgeon, S. L., \& Gauthier, S. F. (2002). Interbiopolymer complexing between $\beta$-lactoglobulin and low- and high-methylated pectin measured by potentiometric titration and ultrafiltration. Food Hydrocolloids, 16(6), 585-591.

Girard, M., Turgeon, S. L., \& Gauthier, S. F. (2003a). Quantification of the interactions between $\beta$-lactoglobulin and pectin through capillary electrophoresis analysis. J. Agric. Food Chem., 51(20), 6043-6049.

Girard, M, Turgeon, S.L, \& Gauthier S.F. (2003b). Thermodynamic parameters of betalactoglobulin-pectin complexes assessed by isothermal titration calorimetry. J Agric Food Chem. 51 (15), 4450-5455.

Girard, M., Sanchez, C., Laneuville, S. I., Turgeon, S. L., \& Gauthier, S. F. (2004). Associative phase separation of $\beta$-lactoglobulin/pectin solutions: A kinetic study by small angle static light scattering. Colloids and Surfaces B: Biointerfaces, 35(1), 15-22.

Gummel, J., Boue', F., Deme', B., Cousin, F. (2006). Charge Stoichiometry Inside Polyelectrolyte -Protein Complexes: A Direct SANS Measurement for the PSSNa -Lysozyme System J. Phys. Chem. B, 110, 24837-24846. 
Kuehner, D. E., Engmann, J., Fergg, F., Wernick, M., Blanch, H. W., \& Prausnitz, M. (1999). Lysozyme Net Charge and Ion Binding in Concentrated Aqueous Electrolyte Solutions. J. Phys. Chem. B, 103, 1368-1374.

Levis, J.C.,Snell, N.S.,Hirschmann, D.,J.\& Fraenkel-Konrat,H. (1950). Amino acid composition of egg proteins. J.Biol. Chem.186,23-36.

Lin, L., Xu W., Liang, H', He, L., Liu, S, Li, Y, Li, B., \& Chen, Y. (2015). Construction of pHsensitive lysozyme/pectin nanogel for tumor methotrexate delivery.Colloids Surfaces B: Biointerfaces, 126, 459-466.

Maroziene, A., \& De Kruif, C.G. (2000). Interaction of pectin and casein micelles. Food Hydrocolloids, 14 (4), 391-394.

May, C. D. (1990). Industrial Pectins: Sources, Production and Applications. Carbohydrate Polymers 12, 79-99.

Michaels, A. S., Mir, L., \& Schneider, N. S. (1965a). A conductometric study of polycation-polyanion reactions in dilute solution. J. Phys. Chem., 69, 1447-1455.

Michaels, A. S., Falkenstein, G. L.,\& Schneider, N. S. (1965b). Dielectric properties of polyanion-polycation Ccmplexes. J. Phys. Chem., 69, 1456-1465.

Ngouemazong, D.E., Tengweh, F. E., Duvetter, T., Fraeye, I., Van Loey, A., Moldenaers, P., \& Hendrickx, M. (2011). Quantifying structural characteristics of partially de-esterified pectins. Food Hydrocolloids, 25, 434-443.

Oulahal, N., Degraeve, P., \& Gharsallaoui, A. (2014). Properties of lysozyme/low methoxyl (LM) pectin complexes for antimicrobial edible food packaging. Journal of Food Engineering 131, 18-25.

Parmar, A. S., \& Muschol, M.(2009). Hydration and hydrodynamic interactions of lysozyme: Effects of chaotropic versus kosmotropic ions. Biophys. J., 97, 590-598.

Plashchina, I. G., Zhuravleva, I. L., \& Antonov, Y.A. (2007). Phase behavior of gelatin in the presence of pectin in water-acid medium. Polymer Bulletin, 58, 587-595.

Rezwan, K., Meier, L. P., \& Gauckler, L. (2005). Lysozyme and bovine serum albumin adsorption on uncoated silica and $\mathrm{AlOOH}$-coated silica particles: the influence of positively and negatively charged oxide surface coatings. J. Biomaterials, 26, 43514357.

Ru, Q. M., Wang, Y. W., Lee, J. Y., Ding, Y. T., \& Huang, Q. R. (2012). Turbidity and rheological properties of bovine serum albumin/pectin coacervates: effect of salt concentration and initial protein/polysaccharide ratio. Carbohydrate Polymers, 88, 838-846. 
Saravanan, M. (2010). Pectin-gelatin and alginate-gelatin complex coacervation for controlled drug delivery: Influence of anionic polysaccharides and drugs being encapsulated on physicochemical properties of microcapsules. Carbohydrate Polymers 80 (3), 808-816.

Schmidt, I., Cousin, F., Huchon, C., Boué, F., \& Axelos, M.A.V. (2009). Spatial structure and composition of polysaccharide-protein complexes from small angle neutron scattering. Biomacromolecules, 10, 1346-1357.

Serov, A.V., Antonov, Y.A., \& Tolstoguzov, V.B. (1985). Isolation of lactic whey proteins in the form of complexes with apple pectin. Die Nahrung, 29, 1, 19-30.

Seyrek, E., Dubin, P. L., Tribet, C., \& Gamble, E. A. (2003). Ionic strength dependence of protein-polyelectrolyte interactions. Biomacromolecules, 4, 273-282.

Sophianopoulos, A. J., \& Vanholde, K. J. (1961). Evidence for dimerization of lysozyme in alkalin solution. Biol. Chem., 236, PC82-PC83.

Sperber, B. L. H. M., Schols, H. A., Stuart, M. A. C., Norde, W., \& Voragen, A. G. J. (2009). Influence of the overall charge and local charge density of pectin on the complex formation between pectin and beta-lactoglobulin. Food Hydrocolloids 23, 765-772.

Sperber, B. L. H. M., Stuart, M. A. C., Schols, H. A., Voragen, A. G. J, \& Norde, W. (2010). Overall Charge and Local Charge Density of Pectin Determines the Enthalpic and Entropic Contributions to Complexation with $\beta$-Lactoglobulin. Biomacromolecules, $11(12), 3578-3583$.

Takahashi, D., Kubota, Y., Kokai, K., Izumi, T., Hirata, M., \& Kokufuta, E. (2000). Effects of surface charge distribution of proteins in their complexation with polyelectrolytes in an aqueous salt-free system. Langmuir, 16, 3133-3140.

Tolstoguzov, V. B. (1986). In J. R. Mitchell \& D. A. Ledward, Functional properties of food macromolecules, (pp. 385-415).

Tolstoguzov, V.B. (1991), Functional Properties of food proteins and role of protein polysaccharide interactions. Food Hydrocolloids, 4, 429-468

Tolstoguzov, V.B. (2003). Some thermodynamic considerations in food formulation. Food Hydrocolloids, 17(1), 1-23.

Tsuboi, A., Izumi, T., Hirata, M., Xia, J., Dubin, P. L., \& Kokufuta, E. (1996). Complexation of proteins with a strong polyanion in an aqueous salt-free System. Langmuir, 12, 62956303.

Valstar, A., Brown, W., \& Almgren, M. (1999). Interactions of globular proteins with surfactants studied with fluorescence probe methods Langmuir, 15, 2635-2643. 
607

608

609

610

611

\section{Captions to figures}

613 Figure 1. The turbidity values at $500 \mathrm{~nm}\left(\tau_{500}\right)$ as a function of the pectin/lys weight ratio q for P16.2/lys

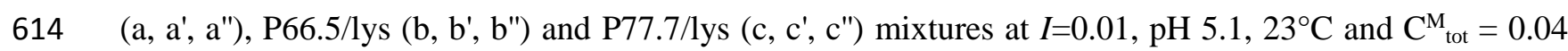
615

Properties of beta-Lactoglobulin/Pectin Coacervates: Effects of Added Salt and Initial Protein/Polysaccharide Ratio. Biomacromolecules, 8, 992-997.

Zaleska, H., Ring, S., \& Tomasik, R. (2000). Apple pectin complexes with whey protein. Food Hydrocolloids, 14, 377-382. $w t \%$. In the insets (a', a", b', b", c', c" ) qonset and q $\mathrm{q}_{\text {set }}$ indicate the transitions between no complex formation and formation of soluble complexes whereas $\mathrm{q}_{\phi}$ and $\mathrm{q}_{\phi}{ }_{\phi}$ indicate the transitions between formation of soluble and insoluble complexes.

Figure 2. (a) $\mathrm{q}_{\text {Max }}$ and $1 / \mathrm{q}_{\mathrm{Max}}$ as a function of DM. (b) The qOnset (circles) and $\mathrm{q}_{\phi}$ (stars) values of pectin/lys systems as a function of DM. (c) The zeta potential $(\zeta)$ as a function of the weight ratio $\mathrm{q}$ for pectin/lys systems, $\mathrm{C}^{\mathrm{M}}{ }_{\text {tot }}=0.3 \mathrm{wt} \%$.

Figure 3. The yield of lys in the complex phase and supernatant of the pectin/lys systems as a function of $\mathrm{q}$ for P16.2/lys (a), P38.2/lys (b), P66.5/lys (c). pH 5.1, $I=0.01,23^{\circ} \mathrm{C}$ and $\mathrm{C}^{\mathrm{M}}{ }_{\text {tot }}=0.3 \mathrm{wt} \%$.

Figure 4. (a) The yield of lys in the complex phase and supernatant of the pectin/lys systems as a function of q for P77.7/lys systems, (b) The maximum yield ( $\left.\mathrm{Y}_{\max }\right)$ of lys in the complex phase for pectin/lys systems as a function of DM. pH 5.1, $I=0.01,23^{\circ} \mathrm{C}$ and $\mathrm{C}^{\mathrm{M}}{ }_{\text {tot }}=0.3 \mathrm{wt} \%$.

Figure 5. Brightfield microscopy images for P16.2/lys (a,c) and P66.5/lys (b,d) systems with increasing total concentration of biopolymers from $0.04 \mathrm{wt} \%(\mathrm{a}, \mathrm{b})$ to $0.4 \mathrm{wt} \%(\mathrm{c}, \mathrm{d}) . \mathrm{q}=\mathrm{q}_{\mathrm{Max}}$, full length of images is $170 \mu \mathrm{m}$. (e) SEM image of the P66.5/lys system. $\mathrm{C}_{\mathrm{lys}}=10^{-5} \mathrm{wt} \%, \mathrm{q}=0.03$. A indicates a nucleus of the complex phase, B indicates small associates of a few nuclei, $\mathrm{C}$ indicates large complex associates before droplet formation. $\mathrm{pH} 5.1, I=0.01$ and $23^{\circ} \mathrm{C}$.

Figure 6. The intensity size distribution as a function of weight ratio q for $(\mathrm{a}, \mathrm{b}) \mathrm{P} 16.2 / \mathrm{lys}$ system. $\mathrm{C}^{\mathrm{M}}$ tot $=$ $0.04 \mathrm{wt} \%$. pH $5.1, I=0.01$ and $23^{\circ} \mathrm{C}$.

Figure 7. The intensity size distribution as a function of weight ratio q for (a,b) P38.2/lys system and (c) P66.5/lys system, $\mathrm{C}^{\mathrm{M}}{ }_{\text {tot }}=0.04 \mathrm{wt} \%$. pH 5.1, $I=0.01$ and $23^{\circ} \mathrm{C}$.

Figure 8. (a) Turbidity values at $500 \mathrm{~nm}\left(\tau_{500}\right)$ of pectin/lys systems as a function of $\mathrm{pH}$ for pectin with different $\mathrm{DM}$ at $\mathrm{I}=0.01$, in the inset $\mathrm{pH}_{\text {Set }}$ as a function of DM (b) Turbidity values at $500 \mathrm{~nm}\left(\tau_{500}\right)$ of pectin/lys systems as a function of ionic strength $(I)$ for pectin with different DM at pH 5.1, in the inset $I_{\text {Set }}$ as a function of DM. $23^{\circ} \mathrm{C}, \mathrm{C}^{\mathrm{M}}$ tot $=0.04 \mathrm{wt} \%, \mathrm{q}=\mathrm{q}_{\mathrm{Max}}$ for $I=0.01$ and $\mathrm{pH} 5.1$ (respectively $0.21,0.24,0.30$ and 0.51 for P16.2, P38.2, P66.5 and P77.7).

Figure 9. Confocal microscopy images of the P16.2/lys mixtures at different q values. a) $q=0.05$; $b$ ) $q=0.25$; c) $\mathrm{q}=0.5$; d) q=0.9; e) $\mathrm{q}=1.5$; f) $\mathrm{q}=2.0$ Full length of images is $1410 \mathrm{~nm}$. Confocal microscopy images of 
642 the P38.2/lys mixtures at different q values. g) q=0.05; h) q=0.25; i) $\mathrm{q}=0.5$; j) q=0.9; k) $\mathrm{q}=1.5$; 1) $\mathrm{q}=2.0$; 643 Full length of images is $1410 \mathrm{~nm}$. pH 5.1, $I=0.01,23^{\circ} \mathrm{C}$ and $\mathrm{C}^{\mathrm{M}}{ }_{\text {tot }}=0.2 \mathrm{wt} \%$.

644 Figure 10. Confocal microscopy images of the P66.5/lys mixtures at different q values for $\mathrm{pH}$ 5.1, $I=0.01$, $64523^{\circ} \mathrm{C}$ and $\mathrm{C}^{\mathrm{M}}{ }_{\text {tot }}=0.2 \mathrm{wt} \%$. a) $\mathrm{q}=0.30$; b) $\mathrm{q}=0.9$; c) $\mathrm{q}=1.5$; d) $\mathrm{q}=2.0$. Full length of images is $1360 \mathrm{~nm}$.

646 Figure 11. a) Schematic representation of the binding mechanism. 1) higher reactivity of pectin chain 647 partially covered with lys 2) higher reactivity of free pectin chain. b) Schematic representation of the 648 complex structure of pectin/lys systems as a function of weight ratio (q).

649 


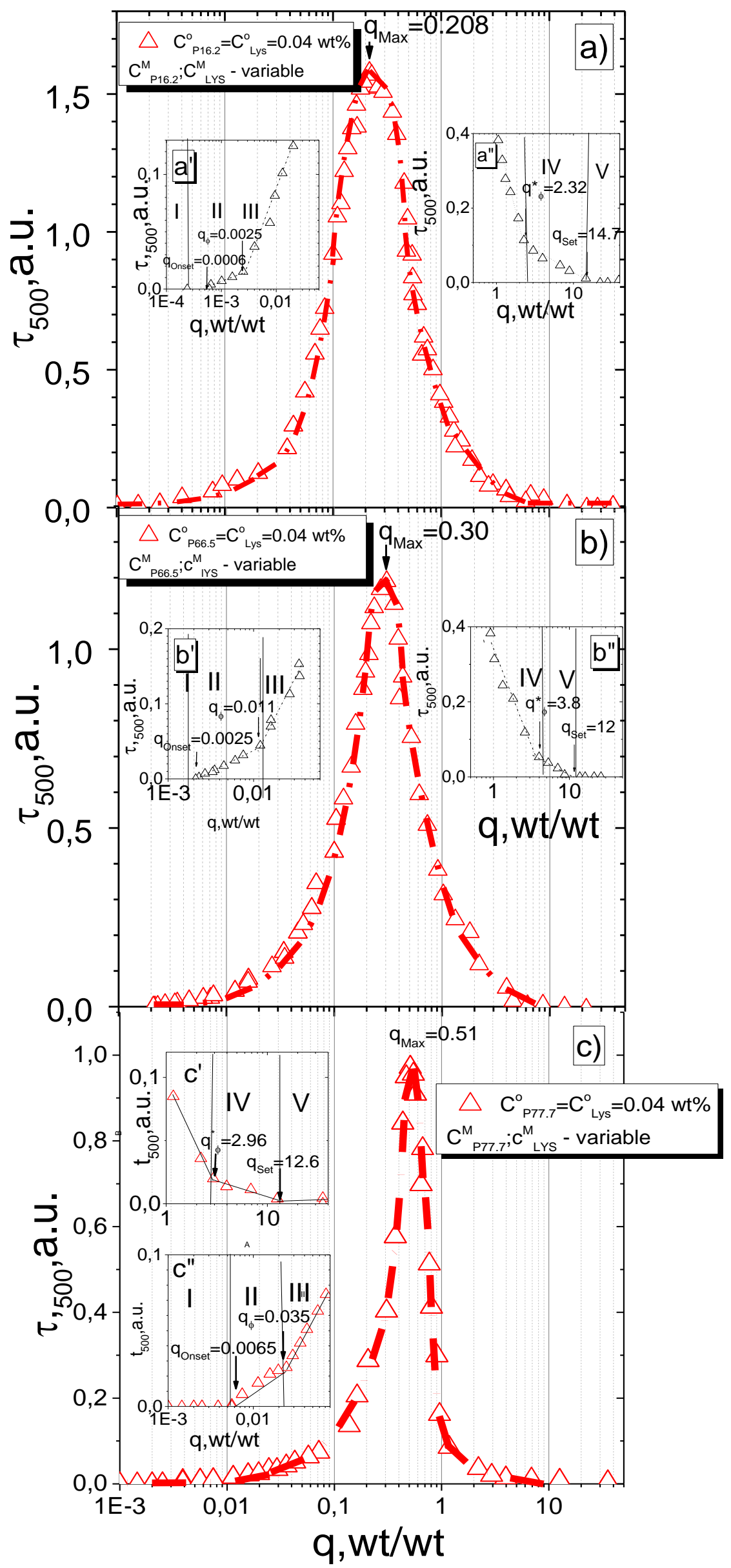

Figure 1 

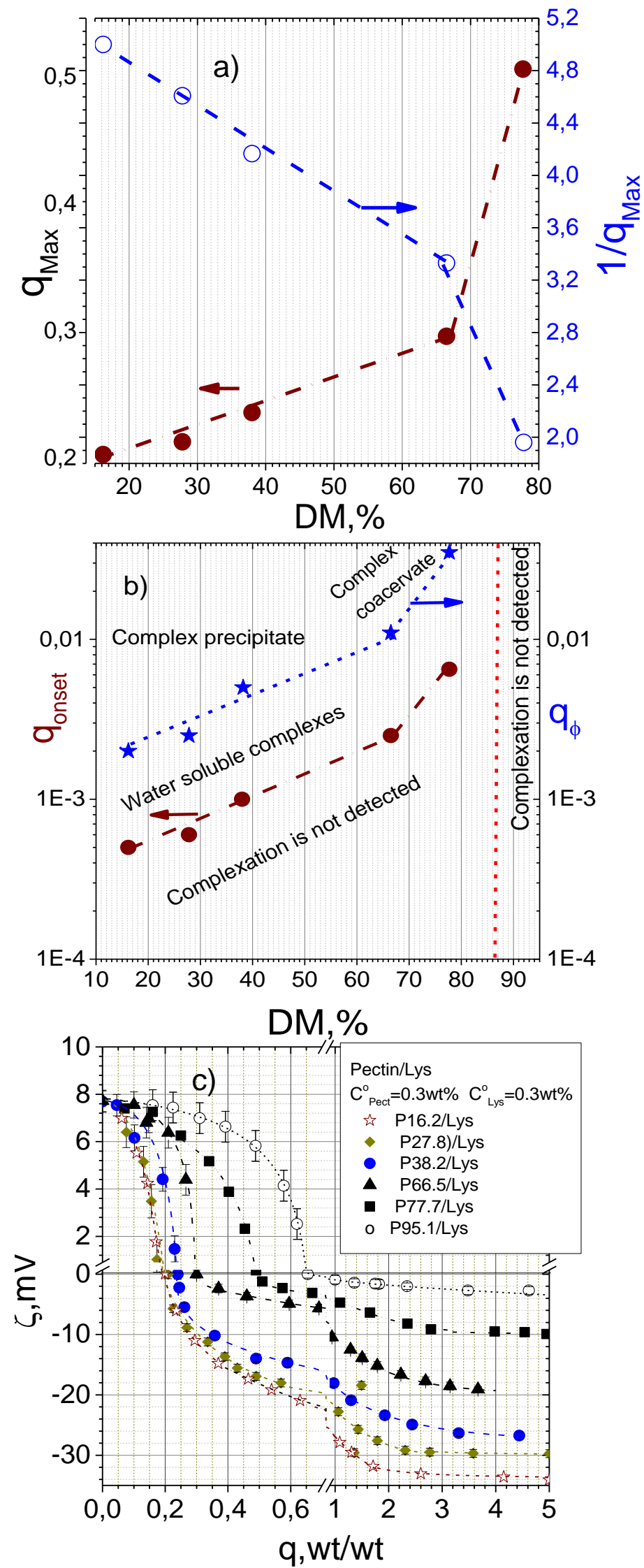

Figure 2 


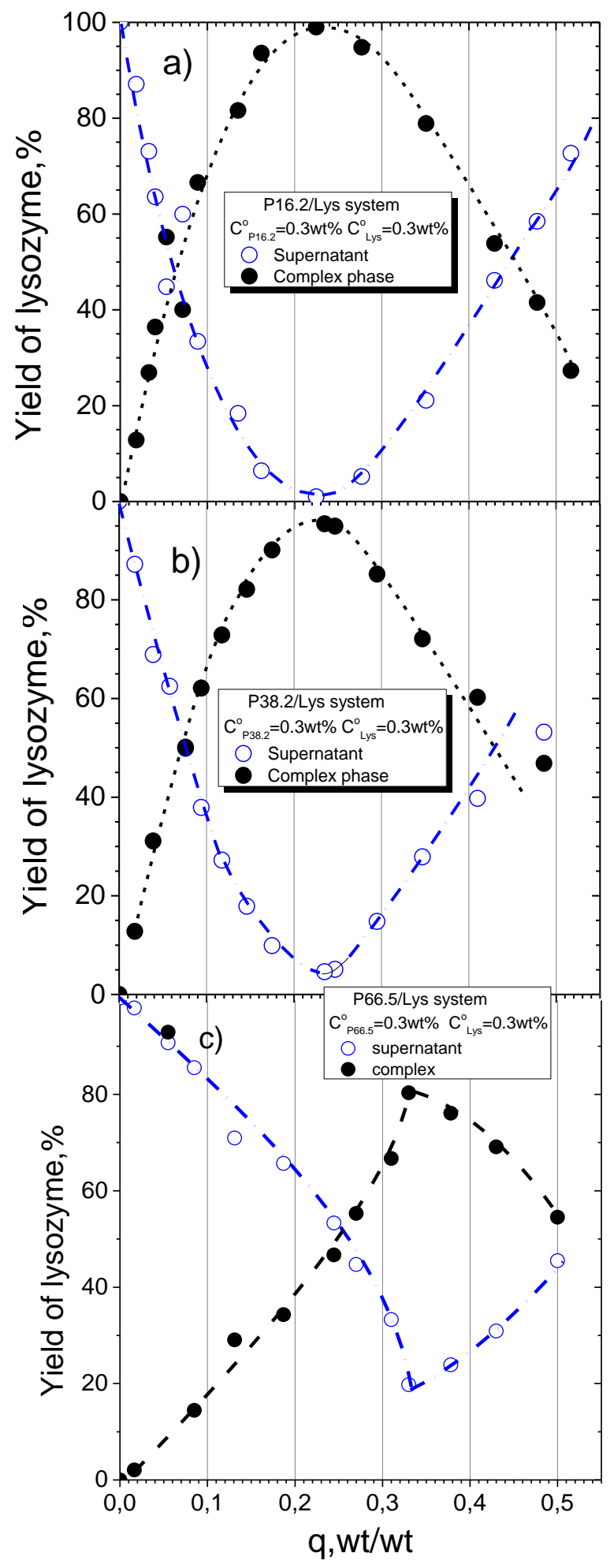

Figure 3 

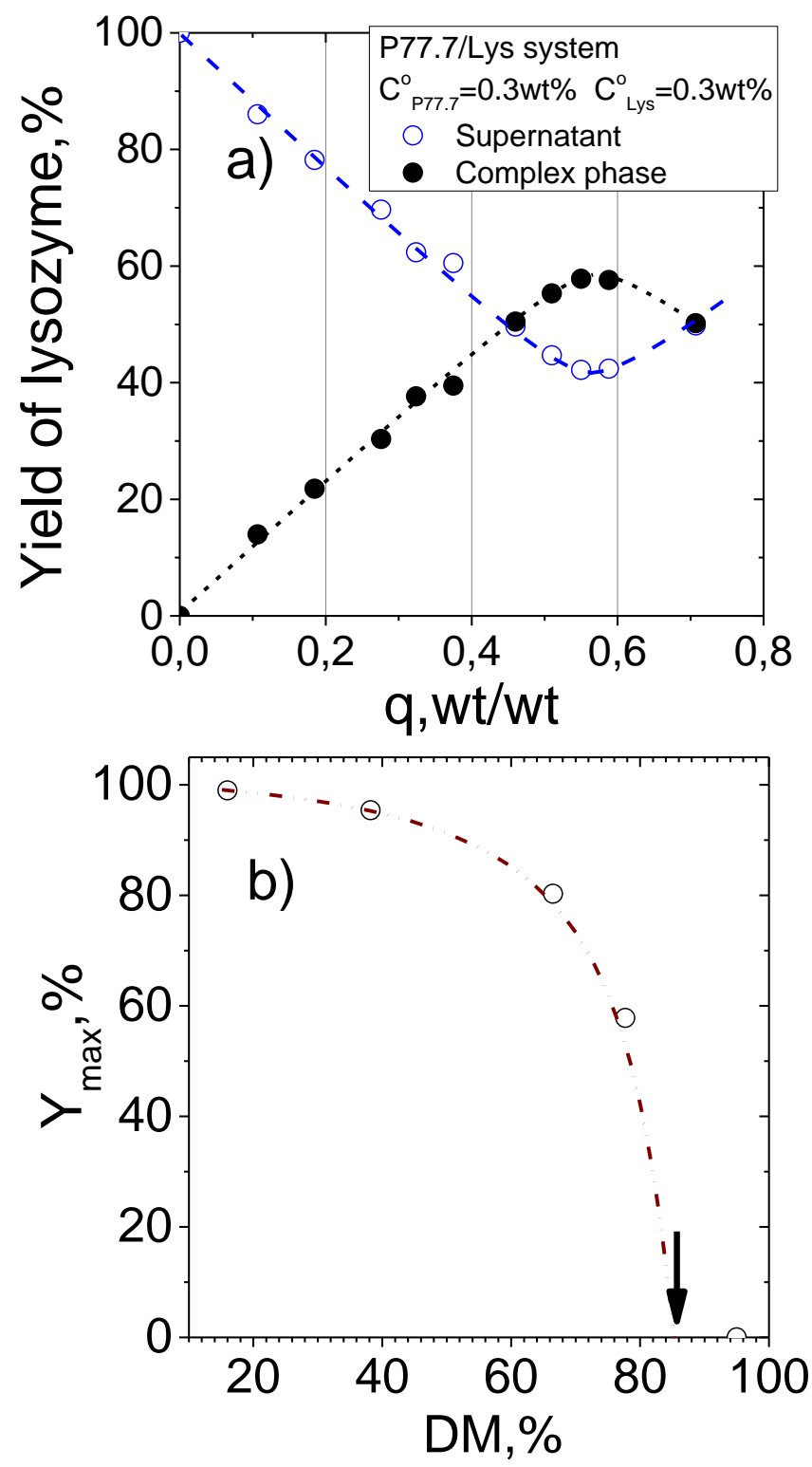

Figure 4 

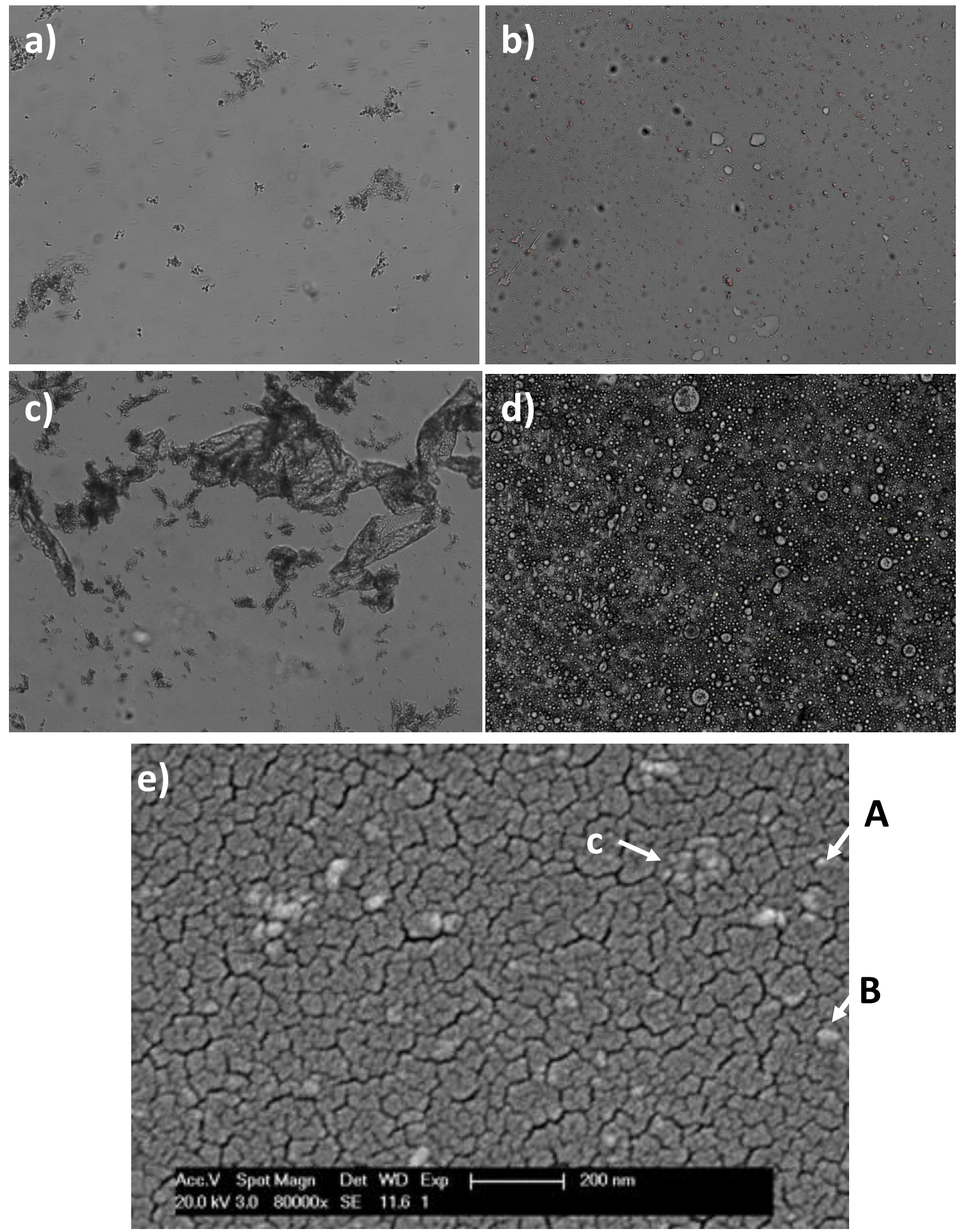

A

B

Figure 5 


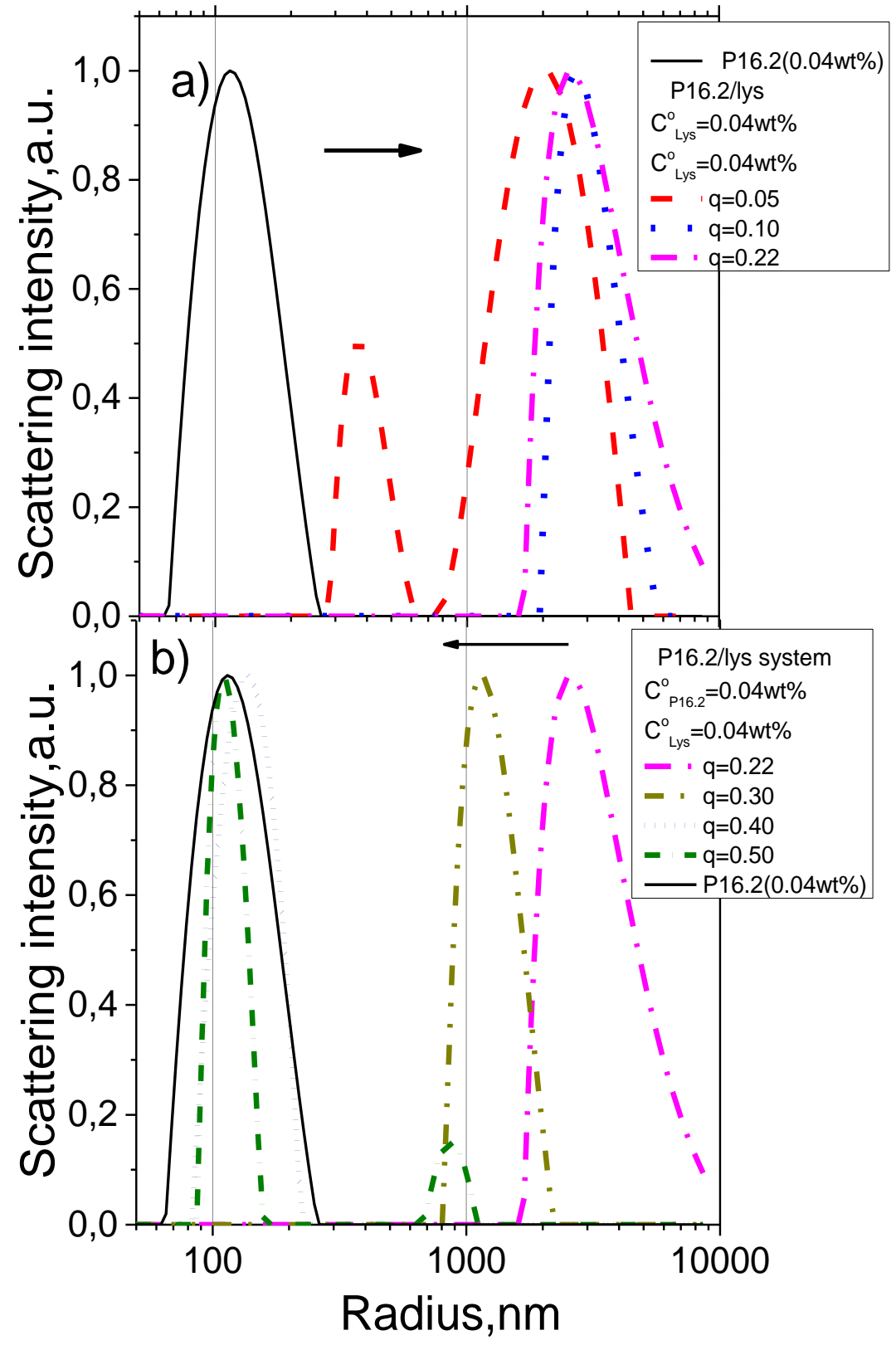

Figure 6 


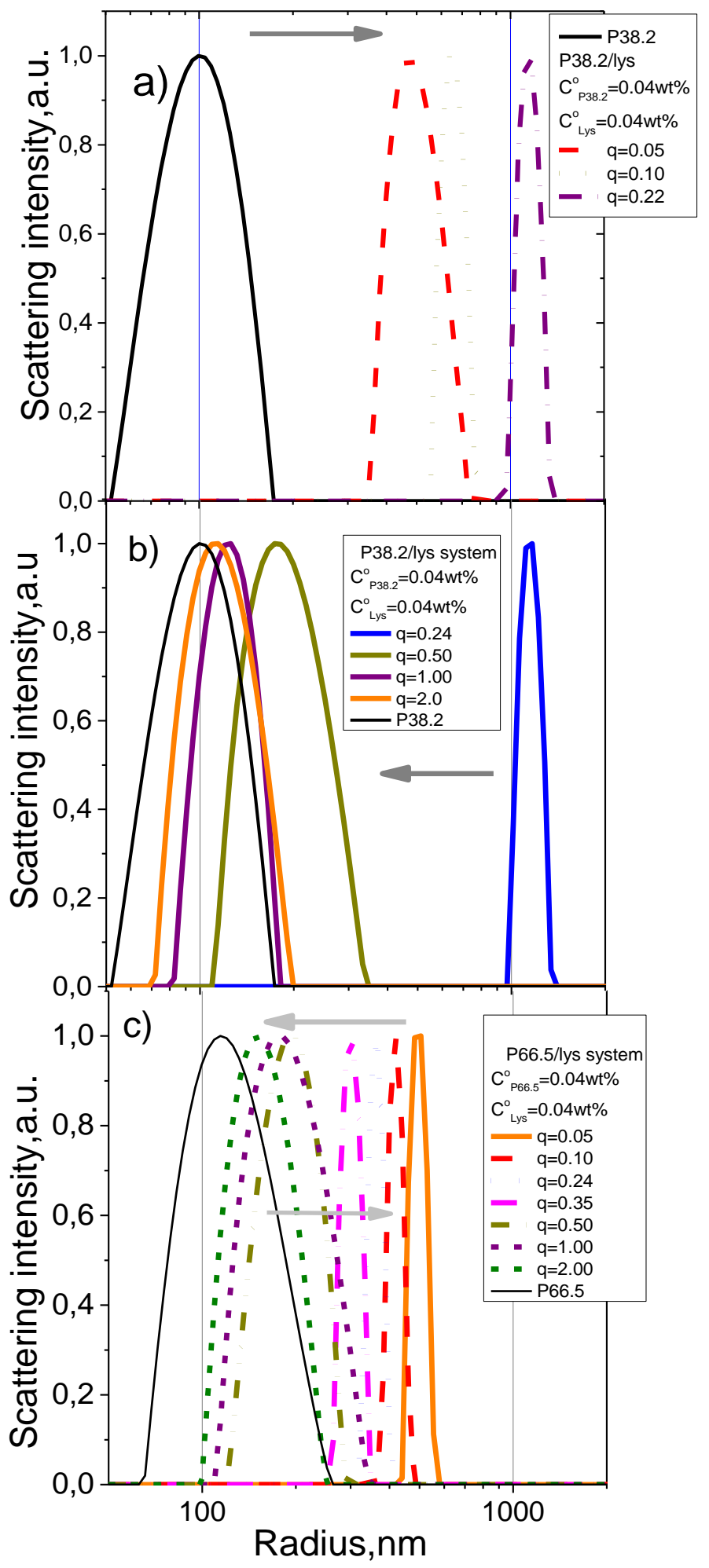

Figure 7 

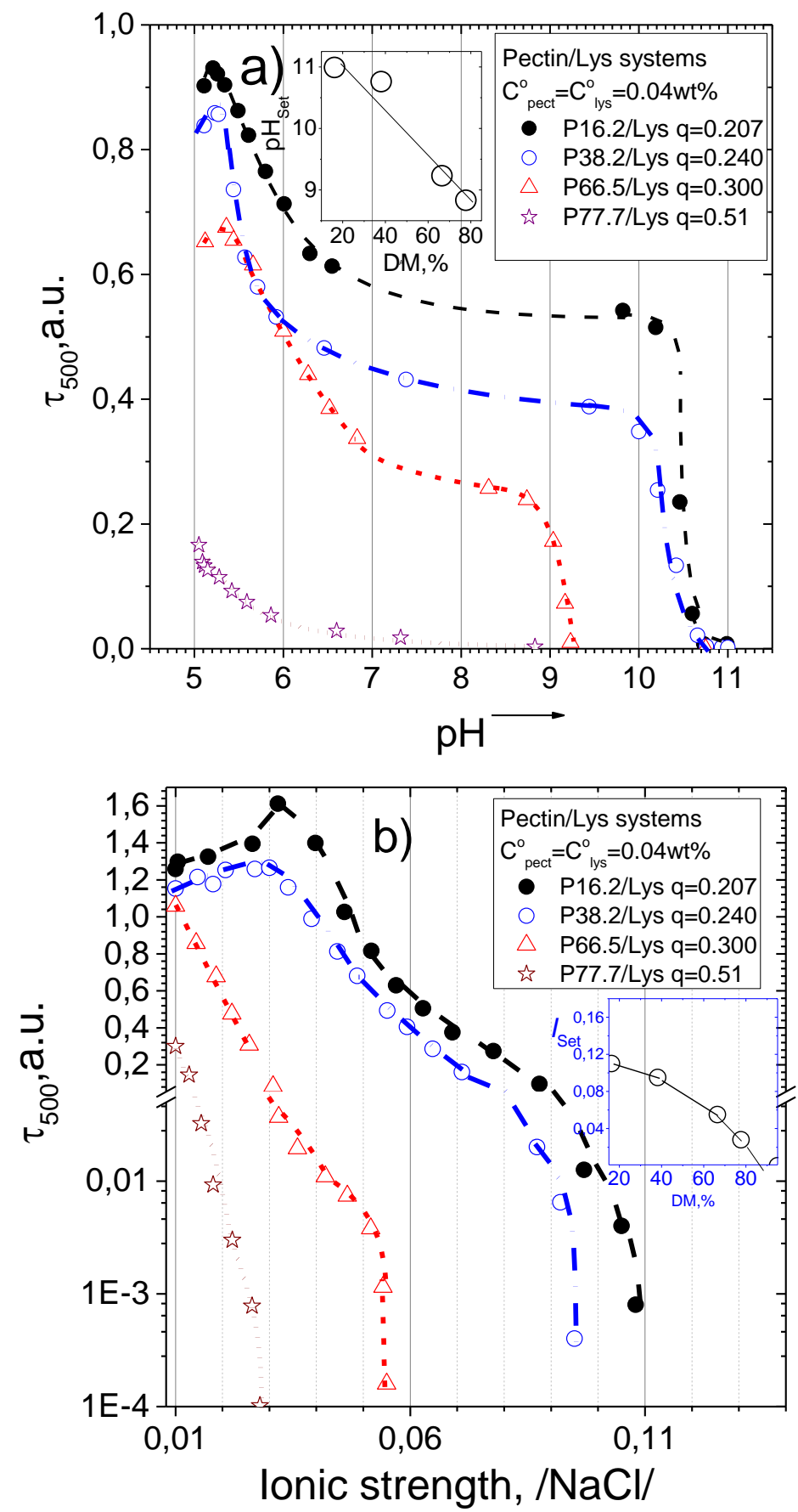

Figure 8 


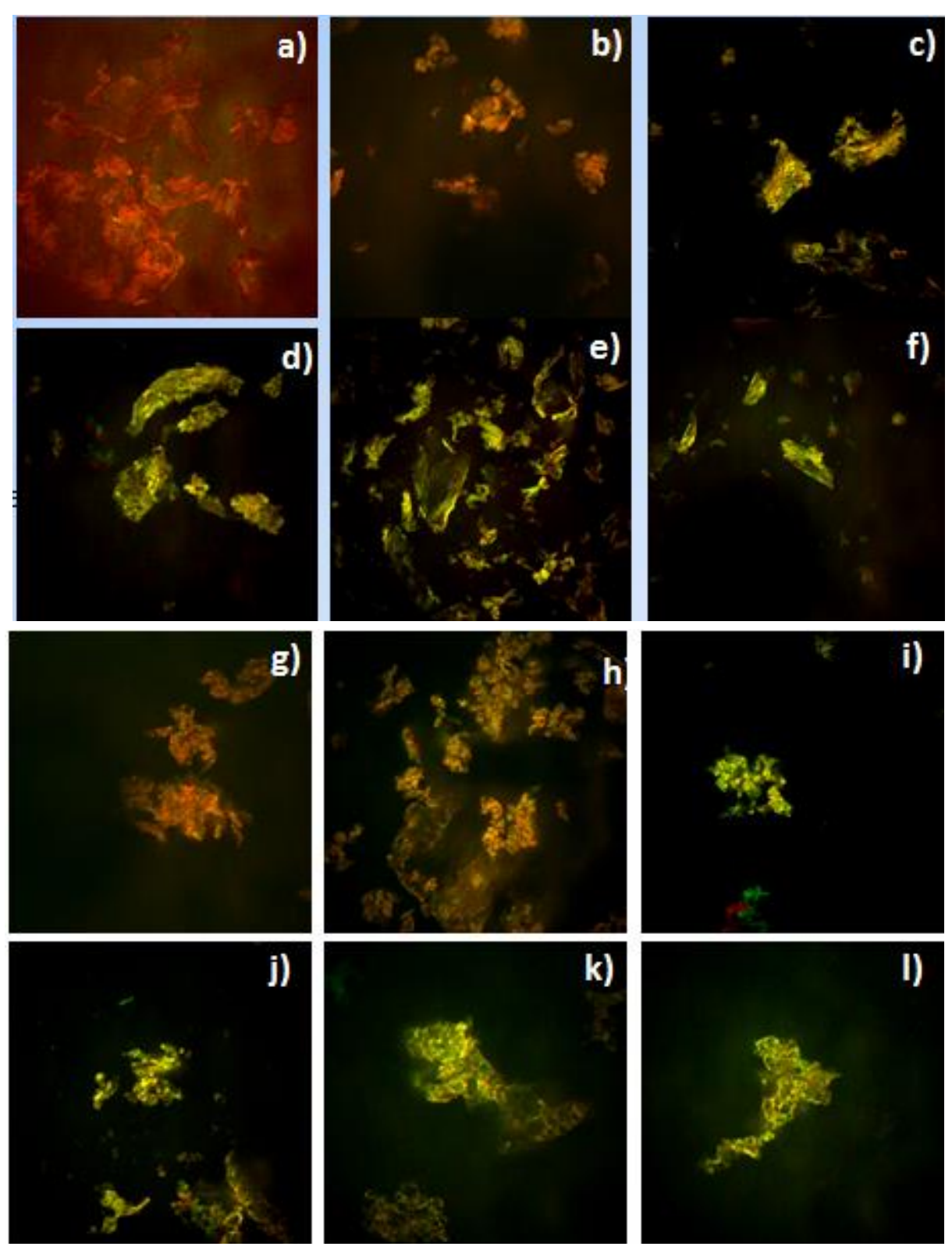

Figure 9 


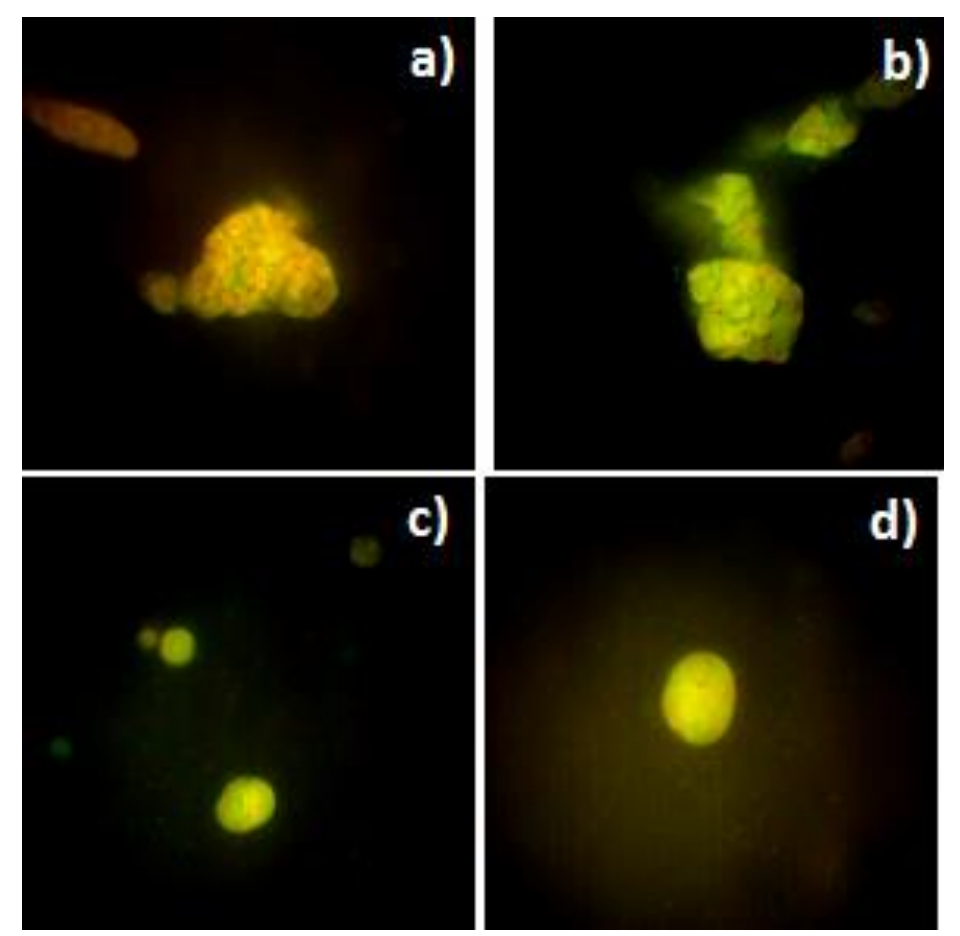

Figure 10 


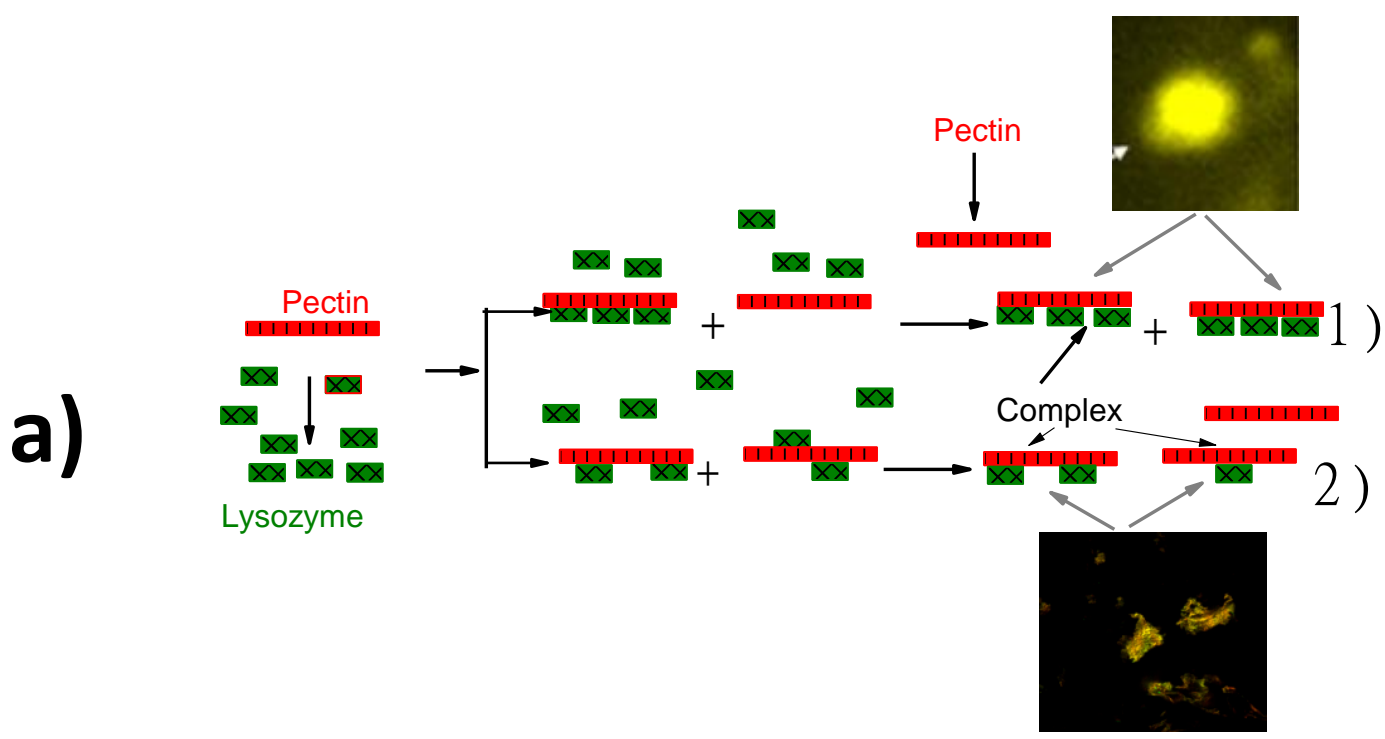

b
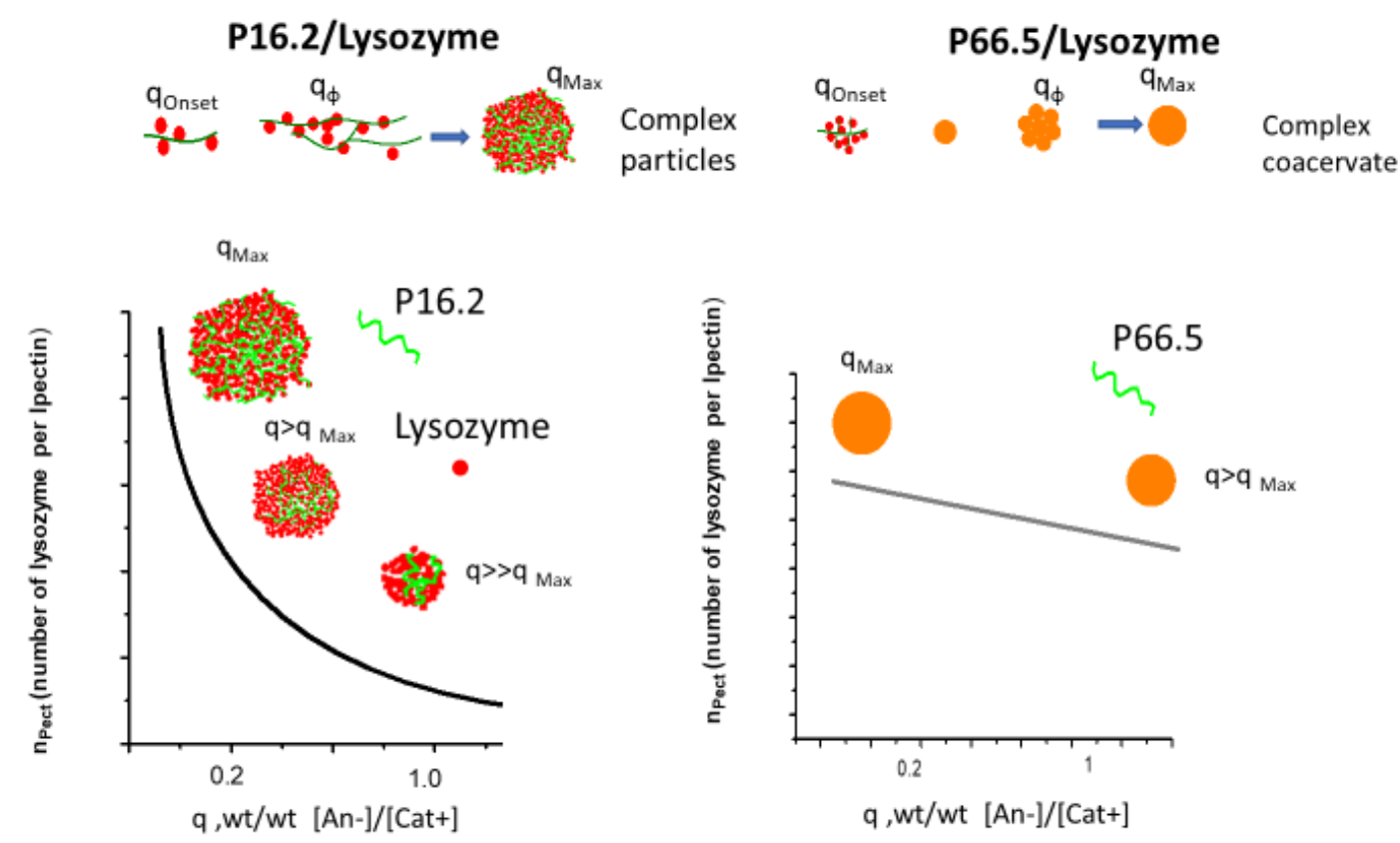

Figure 11 


